\title{
Review of Alpha Adrenoceptor Function in the Eye
}

\author{
D. McAULIFFE-CURTIN, C. BUCKLEY \\ Dublin
}

\begin{abstract}
Summary
The location and function of alpha adrenoceptors in the eye are reviewed with emphasis on pharmacological agents and their role in the management of chronic simple glaucoma.
\end{abstract}

Alquist's (1948) classification of adrenergic receptors began our understanding of the physiological processes at adrenergic nerve endings. The synthesis, storage, release and inactivation of catecholamines have been extensively investigated, particularly in the cardiovascular and central nervous systems. A galaxy of pharmacological agents have become available as receptor agonists and antagonists to mimic block or modulate transmitter function. In recent years, the possibility of altered responsiveness with age has added another dimension to physiological and pharmacological studies of the autonomic nervous system.

Clinical assessment of the autonomic nervous system has depended largely on the measurement of cardiovascular reflexes. With the development of pupillometry, the iris has emerged as a useful additional model for measurement of autonomic responses. Drugs affecting the autonomic nervous system are widely used in the management of a variety of ocular disorders. However, the physiological mechanisms underlying autonomic function in the eye remain poorly defined and more specific use of pharmacological agents would result from improved understanding. Attention has focused on beta-adrenoceptors since the introduction of ophthalmic beta blockers in 1976. The object of this paper is to discuss the relatively limited amount of data available on the role of alpha-adrenoceptors in the eye.

\section{Alpha adrenoceptors: sites and functions}

Alpha-adrenoceptors can be divided into alpha $_{1}$ and alpha $a_{2}$ subtypes based on the relative potencies of agonist and antagonist drugs and independent of receptor location. Both subtypes are present centrally and peripherally. Postjunctional alpha ${ }_{1}$ and alpha ${ }_{2}$ adrenoceptors mediate contraction of smooth muscle. Prejunctional alpha $\mathrm{a}_{2}$ adrenoceptors are known to inhibit the release of noradrenaline by sympathetic postganglionic fibres. Modulation of neurotransmission by alpha $a_{2}$-adrenoceptors is better defined in peripheral tissues than in the central nervous system. ${ }^{1}$

Alpha-adrenoceptors are widely distributed in the eye,$^{2}$ especially in the smooth muscle cells of the iris, the blood vessels of the conjunctiva as well as those of the ciliary processes and the aqueous outflow tract. The distribution and density of alpha-subtypes in ocular structures, an important determinant of drug response, varies considerably between species. Localisation of receptor sites by binding studies have been largely carried out in animal preparations. Iris-ciliary body prep- 
arations from rabbits have been shown to contain predominantly beta $a_{2}$ adrenoceptors with a large population of alpha $\mathrm{a}_{2}$-adrenoceptors also present. ${ }^{3}$ Beta $_{2}$ adrenoceptors have also been predominantly isolated in cultured human trabecular cells and bovine non-pigmented ciliary epithelial cells. ${ }^{4}$

\section{Alpha-adrenoceptors and the iris}

In the iris, alpha $a_{1}$-adrenoceptors mediate the contraction of the dilator pupillae. Phenylephrine is a selective alpha agonist which results in maximum mydriasis in 60 minutes, ${ }^{5}$ varying in extent with the degree of pigmentation of the iris. ${ }^{6}$ A concentration of 5 per cent phenylephrine is sufficient to achieve a maximum response despite the widespread clinical usage of 10 per cent. ${ }^{7}$ It has been suggested that increased sensitivity of alpha-adrenoceptors accompanies a reduction in sympathetic tone in the elderly. ${ }^{8}$ In a recent study ${ }^{5}$, comparing the mydriatic response to phenylephrine in elderly and young age groups, the maximum dilatation reached was similar in both age groups. When the difference in baseline pupil size was eliminated by the administration of thymoxamine, a selective alpha $_{1}$ antagonist, the mydriatic response was was the same in both groups. While there appears to be a reduced sympathetic dilator tone in the elderly, there is not the increased sensitivity of alpha-adrenoceptors expected with denervation hypersensitivity. The reduction in pupil size associated with age must be caused by either a central effect or a deficiency in a key intracellular coupling step beyond the alpha $_{1}$ receptor. Investigations in the rabbit iris-ciliary body suggest that alterations in the phosphoinositide hydrolysis system and its derived second messenger may explain altered responses to alpha $a_{1}$-adrenoceptor stimulation. ${ }^{9}$

Measurement of pupil size following the administration of alpha $a_{2}$ agonists has yielded conflicting results.

The traditionally used alpha $a_{2}$ agonist clonidine is relatively non-selective and acts both centrally and peripherally. Given intravenously, clonidine has been reported to produce a dose dependent mydriasis in cats and rats, reversed by the alpha $a_{2}$ antagonist yohimbine.$^{10}$ In human subjects, clonidine $(0.25$ per cent) applied topically also produced mydriasis, probably by a direct action on alpha ${ }_{2}$-adrenoceptors in the iris. ${ }^{11}$ Clonidine given intravenously to healthy volunteers in low doses of $0.1 \mathrm{mg}$ and $0.2 \mathrm{mg}$, was found to cause miosis reversed by the alpha $\mathrm{a}_{2}$ antagonist RX 781094. ${ }^{12}$ It is likely that at this dose, clonidine is acting centrally to reduce sympathetic tone to the iris, thus producing parasympathetic dominance and pupillary constriction. Antagonism of the miotic action of clonidine by RX 781094 may also be at centrally located alpha-adrenoceptors. Newer selective alpha $a_{2}$ agonists such as B-HT920 topically applied in rabbits, produced no change in pupil size. ${ }^{13}$ The absence of mydriasis with these newer agents has created an interest in the role of alpha stimulating drugs as ocular hypotensive agents.

\section{Alpha adrenoceptors and retinal blood vessels}

The blood vessels of the retina and optic disc autoregulate in order to maintain an almost constant blood flow despite changes in arterial or intraocular pressure. ${ }^{14}$

The tissue levels of oxygen and carbon dioxide $_{2}$ are mainly responsible for this autoregulation. However, retinal vessels have been shown to have specific binding sites for the binding of catecholamines and angiotensin. ${ }^{14}$

These specific adrenergic binding sites must be located to understand if they sites are functional receptors that help maintain haemodynamic homeostasis, and to determine if their stimulation modifies the autoregulatory capabilities of these vessels under physiological or pathological circumstances. Limitation of autoregulation may be the reason that some individuals suffer glaucomatous optic nerve damage while others with intact regulation may not suffer damage.

\section{Alpha-adrenoceptors and aqueous humour dynamics}

Intraocular pressure is determined by the balance between inflow and outflow of aqueous humour ${ }^{2}$. Effects of phenylephrine (alpha ${ }_{1}$ agonist), prazosin (alpha ${ }_{1}$ antagonist), clonidine (alpha $a_{2}$ agonist), and yohimbine 
(alpha ${ }_{2}$ antagonist), on aqueous humour dynamics have been studied with a cat eye model. ${ }^{15}$ Phenylephrine inhibited outflow more than formation, causing an ocular hypertensive effect. Prazosin suppressed formation more than outflow. However, its effect on outflow is sufficient to limit its usage as a clinically effective ocular hypotensive agent. Both clonidine and yohimbine influenced aqueous humour formation more than outflow and thus lowered intraocular pressure. The conventional theory of receptor antagonism did not function at alpha ${ }_{2}$-receptor sites in the cat model. In the rabbit, stimulation of the alpha $a_{2}$ subtype with the selective agonist B-HT920 resulted in a decreased intraocular pressure which was blocked by yohimbine. ${ }^{13}$ Topical prazosin has also been shown to lower intraocular pressure in rabbits, an effect independent of the effect on systemic blood pressure. ${ }^{16}$

While the systemic effects of clonidine and prazosin limit their use in the eye, pharmacological studies are aimed at identifying dominant receptors at both inflow and outflow tracts. The development of selective alpha $_{2}$ agonists may yield a new class of antiglaucomatous drugs, free of mydriatic response and systemic side effects. The alpha agonist phenylephrine has been shown to produce a biphasic effect on intraocular pressure an initial rise followed by a late occurring fall. ${ }^{17}$ Phenylephrine is not used clinically in man as an ocular hypotensive agent because of the risk of acute angle closure glaucoma. The alpha $_{1}$ antagonist thymoxamine, however, is used in the management of acute glaucoma, lowering intraocular pressure by overcoming pupil block. ${ }^{18}$

\section{Conclusion}

Animal models have been used in pharmacological investigations that are not permissible in the human eye. The disadvantage of most animal models, with the exception of sub-human primates, is the variation in population and distribution of receptor subtypes, and the differences in anatomy of the irido corneal angle.

Clinical assessment of autonomic function includes pupillometry, which can provide some answers in relation to the neural control of the iris and aqueous flurophotometry which has been used in the study of aqueous humour dynamics. However, the physiological mechanisms underlying autonomic function in the eye remain poorly defined.

Current interest in adrenergic agents has focused on the management of chronic simple glaucoma. Many adrenergic agents provide a significant clinical reduction of intraocular pressure. Both alpha ${ }_{2}$ agonist and antagonist drugs have been shown to lower intraocular pressure. The same paradox exists with beta receptors as both beta agonists and antagonists lower intraocular pressure. Early studies of the ocular response to adrenergic agents were done with drugs having relatively poor receptor specificity. ${ }^{19}$ Adrenaline, for example, stimulates both alpha and beta adrenergic receptors and shows little selectivity for pre- or post-synaptic sites. In addition to nonselectivity, ocular absorption, intraocular drug distribution and metabolism must influence the response to adrenergic agents.

Precise identification, localisation and characterisation of the receptors involved in aqueous humour dynamics are prerequisites to further studies of biochemical cellular events. The receptor is only the initial interface of drug action and transient events at this level initiate further intracellular responses.

Anatomical localisation of receptors in man has recently advanced using membranes prepared by isopycnic centrifugation of tissue homogenates and by slide mounted sections of human trabecular meshwork with in vitro labelling light microscopic autoradiography. These studies have demonstrated predominantly beta $_{2}$ adrenergic receptors in human iris ciliary body preparations, ${ }^{20}$ and trabecular endothelial cells. ${ }^{21}$

At the present time, the quantitative distribution and subtype of alpha receptors in the intraocular muscle fibres, the blood vessels or other cell types in the iris ciliary body has not been established in man or in animals. ${ }^{22}$

It is likely that alpha antagonists can involve different receptor mechanisms to affect the regulation of aqueous humour dynamics. The reduction in intraocular pressure following their administration may result from a decreased rate of aqueous humour formation, an increase in outflow facility or an increase in 
uveoscleral outflow. This multiplicity of sites of action for alpha adrenergic drugs makes detailed analysis of their actions in the iris ciliary body particularly difficult.

There is speculation that the proportion of the two alpha subtypes could vary in different ocular vascular beds and between species. Ciliary process haemodynamics are thought to be modulated primarily by alpha ${ }_{1}$ adrenergic receptors in humans, while in the rabbit iris ciliary body, the large number of alpha adrenergic receptors probably mediate vascular tone. ${ }^{23}$

Vascular post-synaptic alpha 2 receptors cause vasoconstriction by a calcium dependent mechanism when activated by endogenous agonists. Blockade of such receptors could cause a local vasodilatation of blood vessels in the eye, thus increasing outflow. However, vasodilatation of vessels in the ciliary processes could lower ultrafiltration rate which could account for a decrease in aqueous formation.

Additionally, alpha ${ }_{2}$ receptors are negatively coupled to adenylate cyclase in some tissues and can affect both fluid absorption and secretion. It is possible that the secretory capacity of the ciliary epithelium could be directly modulated by the presence of alpha receptors on these cells. This represents a second possible ciliary site of action for alpha $\mathrm{p}_{2}$ selective drugs which is quite separate from the vascular sites in the ciliary processes.

The mechanism for an increase in uveoscleral outflow by drugs with selectivity for both alpha $a_{1}$ and alpha $a_{2}$ receptors might involve simultaneous antagonism of receptors on intraocular muscle cells and on blood vessels.

Studies of alterations of receptor concentration with light microscopic autoradiography in glaucoma and ageing could provide further important information in designing new drugs for the treatment of glaucoma. Topically applied alpha ${ }_{2}$ antagonists may in the future provide high selectivity with fewer systemic side effects in the reduction of intraocular pressure.

\section{References}

${ }^{1}$ Langer SZ and Hicks PE: Physiology of the sympa- thetic nerve ending. Br J Anaesth 1984, 56: 68999.

2 Potter DE: Adrenergic pharmacology of aqueous humor dynamics. Pharmacol Reviews 1981, 33: 133-52.

${ }^{3}$ Mittag T and Tormay A: Adrenergic receptors in iris/ciliary body: direct ligand binding studies. Invest Ophthalmol Vis Sci (A.R.V.O. Suppl.) 1981, 20: 198.

${ }^{4}$ Bloom E, Polansky J, Zlock D, Woop I, Alvarado J, Zumbrum A: Timolol and epinephrine binding to isolated ciliary epithelium and trabecular cells. Invest Ophthalmol Vis Sci (A.R.V.O. Suppl.) 1981, 20: 66 .

${ }^{5}$ Buckley C, Curtin D MacA, Docherty J, Eustace P: Ageing and alpha adrenoceptors in the iris. Eye 1987, 1: 211-16.

${ }^{6}$ Obianwu $\mathrm{HO}$ and Rand $\mathrm{MJ}$ : The relationship between the mydriatic action of ephedrine and the colour of the iris. Br J Ophthalmol 1965, 49: 264-70.

${ }^{7}$ Haddad NJ, Moyer NJ, Riley FC: Mydriatic effect of phenylephrine hydrochloride. Am J Ophthalmol 1970, 70: 729-33.

${ }^{8}$ Docherty JR and O'Malley K: Ageing and alphaadrenoceptors. Clin Sci 1985, 68 (suppl. 10): 133s-6s.

${ }^{9}$ Yousufzai SYK and Abdel Latif AA: Alpha adrenergic receptor induced subsensitivity and supersensitivity in rabbit iris-ciliary body. Invest Ophthamol Vis Sci 1987, 28: 409-19.

${ }^{10}$ Koss MC: Pupillary dilatation as an index of central nervous system alpha $a_{2}$-adrenoceptor activation. $J$ Pharmacol Methods 1986, 15: 1-19.

"Jahnke R and Thumm HW: The influence of Clonidine on eye pressure and pupil dilation. Klin Mbl Augenheilk 1972, 161: 78-82.

${ }^{12}$ Clifford JM, Day MD, Orwin JM: Reversal of Clonidine induced miosis by alpha-adrenoceptor antagonist RX 781094. Br J Clin Pharmacol 1982, 14: 9-101.

${ }^{13}$ Innemee HC, de Jonge A, Van Meel J, Timmermans PB, van Zwieten PA: The effect of selective Alpha ${ }_{1}$-adrenoceptor and alpha ${ }_{2}$-adrenoceptor stimulation on intraocular pressure in the conscious rabbit. Arch Pharmacol 1981, 316: 294-8.

${ }^{14}$ Forster BA, Ferrari-Dileo G, Anderson DR: Adrenergic alpha and alpha $a_{1}$ and alpha $a_{2}$ binding sites are present in bovine retinal blood vessels. Invest Ophthalmol Vis Sci 1987, 28: 1741-5.

15 Chiou GCY: Effects of alpha and alpha activation of adrenergic receptors on aqueous humor dynamics. Life Sci 1983, 32: 1699-704.

${ }^{16}$ Rowland JM and Potter DE: The effects of topical prazosin on normal and elavated intraocular pressure and blood pressure in rabbits. Eur J Pharmacol 1980, 64: 361-3.

${ }^{17}$ Potter De and Rowland JM: Adrenergic drugs and intraocular pressure: Effects of selective B adrenergic agonist. Exp Eye Res 1976, 27: 615-25.

${ }_{18}$ Rutkowski PC, Fernandes JL, Galia MA, Halasa AH: Alpha adrenergic receptor blockade in the 
treatment of angle closure glaucoma. Trans Am Acad Ophthmol Otolaryngol 1973, 77: 137-40.

${ }^{19}$ Murray DL and Leopold HM: Alpha adrenergic receptors in rabbit eyes. Ocular Pharmac 1985, 1: 3-18.

${ }^{20}$ Wax MB and Molinoff PB: Distribution and poperties of beta adrenergic receptors in human iris ciliary body. Invest Ophthalmol Vis Sci 1987, 28: 420-30.
${ }^{21}$ Jampel HD, Lynch MG, Brown RH, Kuhar MJ, De Souza EB: Beta adrenergic receptors in human trabecular meshwork. Invest Ophthalmol Vis Sci 1987, 28: 772-9.

${ }^{22}$ Mittag TW, Tormay A, Severin C, Podos SM: Alpha adrenergic antagonists: correlation of the effect on intraocular pressure and on alpha ${ }_{2}$ adrenergic receptor binding specificity in the rabbit eye. Exp Eye Res 1985, 40: 591-9. 